\title{
Detection of Linear Polarization from SNR Cassiopeia A at Low Radio Frequencies
}

\author{
Wasim Raja and Avinash A. Deshpande
}

Raman Research Institute, Bangalore, India

We report detection of the weak but significant linear polarization from the Supernova Remnant Cas A at low radio frequencies (327 MHz) using the GMRT. The spectropolarimetric data (16 MHz bandwidth with 256 spectral channels) was analyzed using the technique of Faraday Tomography. Ascertaining association of this weak polarization to the source is non-trivial in the presence of the remnant instrumental polarization $(<1 \%$ in our case) - the expected anti-correlation $\rho_{l p, x}$, between the linear polarized intensity and the soft X-ray counts gets masked by the correlation between the Stokes-I dependent instrumental leakage and the X-radiation that is spatially correlated with Stokes-I, if $\rho_{l p, x}$ is computed naively. Hence, we compute $\rho_{l p, x}$ using pixels within ultra narrow bins of Stokes-I within which the instrumental leakage is expected to remain constant, and establish the anti-correlation as well as the correspondence of this correlation with the mean X-ray profile (Figure 1). Given the angular and RM-resolution in our data, the observed depolarization relative to that at higher frequencies, implies that the mixing of thermal and non-thermal plasma within the source might be occurring on spatial scales $\sim 1000 A U$, assuming random superposition of polarization states.
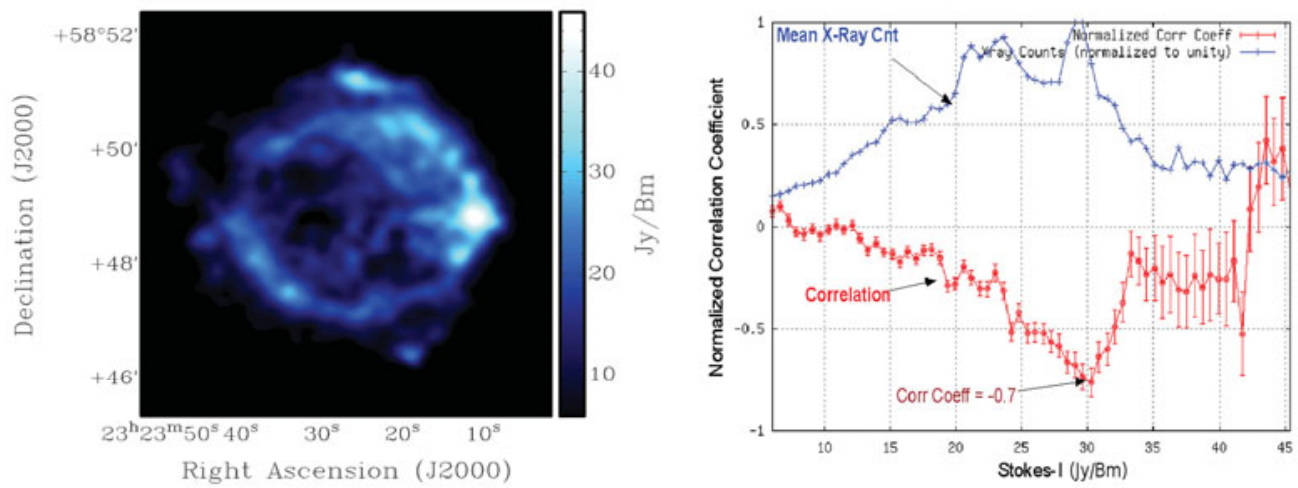

Figure 1. Left: $327 \mathrm{MHz}$ Stk-I image of Cas A. Right: Profile of mean X-ray counts (max normalized to unity) (blue) \& $\rho_{l p, x}$ (at $\mathrm{RM}=-110 \mathrm{rad} / \mathrm{m}^{2}$ ) (red) as a function of binned Stk-I.

\section{References}

Anderson, et al., 1995, ApJ, 441, 300

Brentjens, M. A. \& de Bruyn, A. G. 2005, A\&A, 441, 1217

Burns, B. J. 1966, MNRAS, 133, 67

Downs, G. S. \& Thompson, A. R., 1972, AJ, 77, 120

Ramkumar, P. S. \& Deshpande A. A., 1999, J. Astrophys. Astr., 20, 37 